$12-2019$

\title{
An Approach to Fast Multi-Robot Exploration in Buildings with Inaccessible Spaces
}

Matt McNeill

Damian Lyons

Follow this and additional works at: https://fordham.bepress.com/frcv_facultypubs

Part of the Robotics Commons 


\title{
An Approach to Fast Multi-Robot Exploration in Buildings with Inaccessible Spaces
}

\author{
Matthew McNeill and Damian Lyons
}

\begin{abstract}
The rapid exploration of unknown environments is a common application of autonomous multi-robot teams. For some types of exploration missions, a mission designer may possess some rudimentary knowledge about the area to be explored. For example, the dimensions of a building may be known, but not its floor layout or the location of furniture and equipment inside. For this type of mission, the SpaceBased Potential Field (SBPF) method is an approach to multirobot exploration which leverages a priori knowledge of area bounds to determine robot motion. Explored areas and obstacles exert a repulsive force, and unexplored areas exert an attractive force. While SBPF has advantages over other methods of robot exploration in terms of simplicity and performance, inaccessible space poses a problem: it exerts a permanent attractive force, pulling robots away from useful exploration elsewhere and creating minima at its boundary. Prior research established a simple method of filling in inaccessible space as a solid obstacle once an enclosing boundary is discovered; however, this method requires the entire enclosing boundary to be discovered before it can be filled. In this paper, we propose a novel combined SBPF and frontier-based method of robot exploration called O-SBPF. Our method adds two new space classifications: open, areas known to be accessible; and occluded, areas which may be inaccessible. We describe a ray-casting approach to designate areas as open or occluded, and incorporate this designation into potential vector calculations. We then show the effectiveness of O-SBPF using ROS/Stage in worlds with inaccessible space. O-SBPF significantly outperforms SBPF in rooms with large obstacles, successfully reaching $95 \%$ coverage while SBPF becomes stuck in a minima. In less complex rooms, we show that $O$-SBPF generally reaches $95 \%$ coverage at the same time or before SBPF.
\end{abstract}

\section{INTRODUCTION}

Exploration of unknown environments is a common application of autonomous robots. For some types of mission, including search-and-rescue and C-WMD (Counter-Weapons of Mass Destruction), the most critical goal is the rapid and thorough exploration of all available space. A C-WMD mission may require us to handle a biological, chemical or explosive threat, while a search-and-rescue mission may require us to locate survivors. Both require speed and through exploration. The potential field approach to robot exploration has an advantage in this type of mission due to its simple, uniform control scheme and light computational overhead.

The authors are with the Robotics and Computer Vision Lab, Department of Computer and Information Science, Fordham University, Bronx NY U.S.A. $\{$ mmeneill6, dlyons\} @ fordham.edu
For some missions, a mission designer may possess some rudimentary knowledge about the area to be explored. For example, if exploring an office building, a designer may know the dimensions of the building but not the floor layout or the location of furniture and equipment. The SBPF (SpaceBased Potential Field) method, introduced by Liu and Lyons [14], leverages a priori area bounds information to create a simple potential field control strategy. By exploring within fixed boundaries, unexplored space can exert an attractive force on robots while explored space, obstacles, and other robots exert a repulsive force. SBPF allows as much as an $80 \%$ speedup in area coverage time with the addition of a second robot. Additionally, the SBPF method is able to sidestep local minima situations endemic to the potential field method by increasing the attractiveness of unexplored space as exploration progresses. This change in the potential field over time helps to dislodge robots trapped in a minima.

However, the potential for minima still exists when a building contains inaccessible areas, such as the interior of large columns, inter-floor pipe and cable ducts, or inaccessible outdoor spaces such as courtyards or green spaces. The outer boundary of the inaccessible space can be discovered, but the interior remains unexplored for the duration of the mission. As exploration progresses, robots are increasingly drawn toward the interior of these inaccessible spaces, slowing exploration. Additionally, minima can form at the edge of inaccessible space: surrounding walls push robots away, but the unexplored space inside draws them in. This is a serious constraint for deploying this approach in realistic scenarios.

Liu and Lyons [14] partially address this problem by repeatedly checking accumulated geometric obstacle information for enclosed areas. If such an area is found, it is filled in as a solid obstacle, eliminating the attractive space inside. This solution has two serious drawbacks: one, inaccessible space remains fully attractive until the moment it is no longer justifiable to maintain its attraction; and two, to eliminate the influence of an inaccessible area, robots must discover its entire enclosing boundary in spite of continual attraction toward the interior. The boundaries of some obstacle arrangements may never be fully discovered, jeopardizing both the speed and completeness of the mission.

To retain the strong exploration performance of SBPF but allow it to be used in real buildings with inaccessible space, we introduce a limited frontier to SBPF inspired by [22] through the novel space designations of open and occluded. 
Open spaces are known to be accessible, while occluded spaces may contain inaccessible areas. Like [9], occluded space results from sensor blockage, and represents the unseen space behind obstacles. As in other frontier methods of robot exploration [6] [18], we consider the edge of occluded space an exploration goal. Occluded space edges are incorporated into the sum of attractive forces, encouraging robots to explore its boundary. The remainder of occluded space is neither attractive nor repulsive, removing a major source of distraction from robots as they engage in exploration elsewhere.

With these space designations, we offer two principal contributions: one, our novel method Openness-Enhanced SpaceBased Potential Field (O-SBPF) which leverages occluded designations to create frontiers in potentially inaccessible areas; and two, a ray-casting method leveraging laser sensor range information to determine whether space is open or occluded. We report the results of several experiments in a variety of room configurations to demonstrate the following claims:

1) Some rooms with inaccessible space cannot be fully explored with SBPF, but can be fully explored with O-SBPF.

2) Rooms with inaccessible space that can be fully explored with SBPF can be explored in equal or less time with O-SBPF.

\section{LITERATURE REVIEW}

The SBPF approach to multi-robot exploration was introduced by Liu and Lyons in [14], where it was demonstrated with a simple centralized control mechanism. The approach was extended with a decentralized architecture in [13], where each robot maintains individual accumulated obstacle and exploration information shared through wireless networking. Kenealy proposed further extensions in [10] to aid with the exploration of complex environments, including attraction to narrow openings and a constant "twist" modification to repulsive forces near obstacles.

SBPF is a recent approach to multi-robot exploration. Other methods include frontier-based approaches where the boundary of explored and unexplored space is maintained [5] [16] and decentralized role-based exploration [20]. The application of potential field algorithms to robot exploration is based on a common technique in robot motion planning [4] [11], and hence must also be careful in how it addresses local minima issues [3]. Consequently, it is often combined with other navigation approaches or augmented with techniques to address minima. For example, [15] adapts the potential field approach for UAV (Unmanned Aerial Vehicle) path planning. It addresses local minima with the creation of temporary attractive virtual waypoints, though it requires that robots recognize when they enter a minima. [8] combines traditional pathfinding with potential fields by precomputing a path to a goal around static obstacles, then using potential fields to drive robots away from moving obstacles.

Amigoni [1] placed the decentralized architecture proposed in [13] in a class of communication methods relying on extemporaneous encounters instead of dedicated connections. Other methods of decentralized robot communication in this class include a frontier-based method with UAVs proposed by Cesare [7], which describes various roles UAVs can take on to facilitate communication, including sacrificing themselves in low-battery situations to act as static relays. Additionally, Andre [2] describes an approach for multi-robot exploration with a mechanism for a robot to request assistance from others, specifically to deal with inaccessible space by clearing obstacles blocking entry to the space.

Because all unexplored space is initially attractive in SBPF, the problem of attraction to inaccessible space is likely unique to this approach. However, other approaches to robot exploration and planning must account for obstacles and inaccessible space in various capacities, depending on the specifics of the approach. Wattanavekin [21] proposes an exploration approach where a path is constructed through a set of observation points distributed throughout accessible unexplored space. Like O-SBPF, the detection of an obstacle effects a change in robot behavior, though in this approach it triggers a recomputation of the path. Crucially, an algorithm similar to the inaccessible space detection method in [14] is used to determine if an area is unreachable, and therefore ineligible to contain observation points. Unlike SBPF, the approach does not require recognizing potentially inaccessible areas or eliminating them from consideration because the exhaustive pathfinding will eventually discover all obstacle boundaries.

\section{METHOD}

We begin by briefly reviewing the SBPF method. In SBPF, every point in space within a priori area bounds is initially treated as an attractive goal, drawing robots towards it. Once a point is visited, it becomes repulsive, driving robots away. As in most potential field-based robot navigation methods [18], other robots and obstacles also exert a repulsive force. The summation of these forces at a point in space determines robot heading and speed.

In this paper, we use a modified version of the SBPF potential field equations described in [14]. We consolidate some formulas for legibility in a multiple spatial grid environment, since our method incorporates an additional grid containing the occluded status of each point. Additionally, we simplify repulsive force calculations by eliminating the repulsive force coverage scaling factor $h_{\text {rep }}$ so that repulsion does not shrink as coverage grows. The attractive force coverage scaling factor $h_{\text {att }}$ remains, so attraction continues to grow as coverage grows in order to pull robots toward remaining unexplored areas. Below, sections III-A to III-C 
summarize the SBPF method, while sections III-D onward constitute our contribution.

\section{A. Repulsion}

For a potential field grid $Q$, where each cell $q \in Q$ represents a point in space and contains some value $v(q)$, the repulsive force at cell $q_{i} \in Q$ where $q_{i} \neq q$ caused by the field at $q$ is given by

$$
\begin{gathered}
F_{\text {rep }}\left(q, q_{i}\right)=\left(\frac{\lambda\left(q, q_{i}\right)\left(1-\delta\left(q_{i}\right)\right)}{\rho^{3}\left(q, q_{i}\right)}\right)\left(\frac{1}{\rho\left(q, q_{i}\right)}-\frac{1}{\rho_{0}}\right) \\
F_{\text {rep }}(q, Q)=\sum_{q_{i} \in Q-\{q\}} \begin{cases}F_{\text {rep }}\left(q, q_{i}\right) & \rho\left(q, q_{i}\right) \leq \rho_{0} \\
0 & \rho\left(q, q_{i}\right)>\rho_{0}\end{cases}
\end{gathered}
$$

where

- $\lambda$ is the difference in potential level of the cells, or $v(q)-v\left(q_{i}\right)$.

- $\delta$ is the Dirac delta function, which yields 1 if $q_{i}$ has potential level 0 , and 1 otherwise.

- $\rho\left(q, q_{i}\right)$ is the Euclidean distance from cells $q$ to $q_{i}$.

- $\rho_{0}$ is the maximum repulsion distance.

\section{B. Attraction}

Similarly, attractive force at cell $q \in Q$ is given by

$$
\begin{gathered}
F_{\text {att }}\left(q, q_{i}\right)=h_{\text {att }}(c) \delta\left(q_{i}\right) \lambda\left(q, q_{i}\right) \rho^{2}\left(q, q_{i}\right) \\
F_{\text {att }}(q, Q)=\sum_{q_{i} \in Q-\{q\}} F_{a t t}\left(q, q_{i}\right)
\end{gathered}
$$

where $c$ is the coverage factor, or the fraction of accessible space the robot has visited, and $h_{a t t}(\mathrm{c})$ which is given by

$$
h_{\text {att }}(c)=\frac{k_{a t t}}{c}
$$

where $k_{\text {att }}$ is some constant.

\section{Sum of forces}

We now define three grids: the obstacle grid, occupancy grid, and robot grid, delineated $Q_{o b s}, Q_{o c c}$, and $Q_{b o t}$, respectively. The values contained in each grid are as follows, where $v(q)$ indicates the potential level at point $q$ and $q_{\max }$ is some constant:

- For each $q \in Q_{o b s}, v(q)=q_{\max }$ if the space represented by $q$ is known to contain an obstacle, but is 0 otherwise.

- For each $q \in Q_{o c c}, 0 \leq v(q) \leq q_{\max } . v(q)$ is initially 0 , but is incremented if a robot travels inside.

- For each $q \in Q_{b o t}, v(q)=q_{\max }$ if a robot occupies the space represented by $q$, but 0 is otherwise.

With these grids, we compute a potential vector $F_{\text {total }}$, which determines the direction of a robot within the cell.

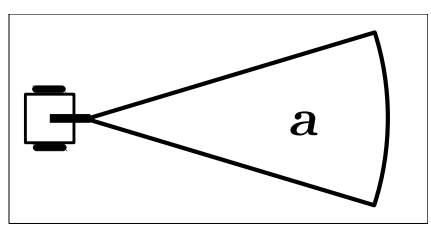

(a) Open space

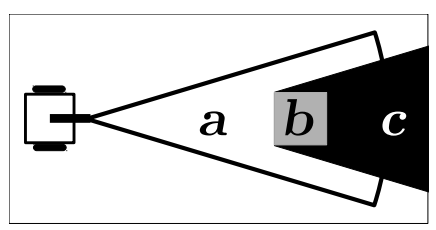

(b) Occluded space
Fig. 1: Open and occluded space derived from laser sensor

The value of $F_{\text {total }}$ is the resultant force at a point $q$ in space due to obstacles, robots, and the space-based fields:

$$
\begin{array}{r}
F_{\text {total }}=w_{\text {obs }} F_{\text {rep }}\left(q, Q_{o b s}\right)+w_{\text {occ }} F_{\text {rep }}\left(q, Q_{o c c}\right) \\
\quad+w_{\text {bot }} F_{\text {rep }}\left(q, Q_{b o t}\right)+w_{\text {att }} F_{\text {att }}\left(q, \delta\left(Q_{o c c}\right)\right)
\end{array}
$$

where $w_{o b s}, w_{o c c}$, and $w_{b o t}$ are weight values for the repulsive forces generated by the obstacle, occupancy, and robot grids, respectively. Similarly, $w_{\text {att }}$ is a weight value for the attractive forces generated by the occupancy grid.

\section{Open and occluded designations}

To improve the speed of exploration and improve coverage in the case of a building with inaccessible areas, our principal contribution to the SBPF method is the enhanced representation of space to include the novel designations of open and occluded. For a potential field grid $Q$, where each cell $q \in Q$ represents a point in space, we designate $q$ as "open" when we know a robot is able to enter $q$. Conversely, we designate $q$ as "occluded" when we do not know if a robot is blocked from entering $q$. We assume a multi-robot team where each robot is equipped with a laser scanner with maximum distance $\rho_{l}$ and a sensing angle of $\theta$. As a practical example, Fig. 1a depicts a robot and the area covered by the range of its sensor, labeled $a$. No obstacle is detected in $a$, so we designate $a$ open space.

Fig. 1b depicts a robot and the area covered by the range of its sensor, labeled $a$, which is again designated open space. However, in this scenario, the sensor detects obstacle $b$ inside $a$. $b$ can be thought to cast a shadow, labeled $c$, from the laser sensor. Because the laser sensor cannot see beyond the obstacle, we do not know if other obstacles exist within $c$, or if the space inside $c$ is accessible at all. Consequently, we designate $c$ occluded space.

We limit open space designations to fall within the laser sensor range $\rho_{l}$ and angle $\theta$. However, we do not constrain occluded space designations by $\rho_{l}$, allowing them to extend to the edge of available space. We justify this approach with the nature of open and occluded designations. An open space designation is absolute, leaving no room for ambiguity: a sensor has scanned the area and found nothing there. Conversely, an occluded space designation implies we do not know whether the space is accessible or not. The presence of an obstacle means we cannot assume that any space behind it is accessible, even space beyond the range of the laser sensor. 


\section{E. Rays and open/occluded grids}

To designate a point in exploration space as open or occluded, we present an approach modeled after the raycasting algorithm [12], [19] simplified for use in a 2D grid environment.

A set of rays $R$ are projected from the robot's location $q$, with a maximum sensor angle $\theta$ in a direction determined by the robot heading $\theta_{\text {robot }}$. The angle of ray $\vec{r}_{i} \in R$, denoted $\theta_{i}$, is given by

$$
\theta_{i}=\theta\left(\frac{i}{n}-0.5\right)+\theta_{\text {robot }}
$$

where $n$ is the number of rays in $R$.

As an initial step, we determine if any rays intersect with an obstacle within sensor range $\rho_{l}$. In this work, we leverage the obstacle grid $Q_{o b s}$, since it is already populated with discovered obstacles. We wish to determine a set of pairs $\left(q_{i}, \vec{r}\right) \in Q_{o b s}^{\perp}$ where $q_{i}$ is a cell in $Q_{o b s}$ containing an obstacle, and $\vec{r}$ is a ray in $R$ which intersects with $q_{i}$. We select pairs for inclusion in $Q_{o b s}^{\perp}$ with the following:

$Q_{o b s}^{\perp}=\left\{\left(q_{i} \in Q_{o b s}, \vec{r} \in R\right) \mid \vec{r} \perp q_{i}, v\left(q_{i}\right)>0, \rho\left(q, q_{i}\right) \leq \rho_{l}\right\}$

where $\vec{r} \perp q_{i}$ indicates whether $\vec{r}$ intersects with $q_{i}, v\left(q_{i}\right)>0$ indicates whether the value at cell $q_{i}$ is larger than 0 and therefore contains an obstacle, and $\rho\left(q, q_{i}\right) \leq \rho_{l}$ indicates whether the distance between the robot at $q$ and cell $q_{i}$ is within the laser sensor distance.

With $Q_{o b s}^{\perp}$, we can designate space as open or occluded. We define new grids $Q_{\text {open }}$ and $Q_{\text {occluded }}$, where all cells in both grids begin with an initial value of 0 . Then, for a given moment of exploration $t$, we assemble intermediary open and occluded grids $Q_{\text {open }}^{t}$ and $Q_{\text {occluded }}^{t}$. The value of a cell $q_{p} \in Q_{\text {open }}^{t}$ is set to 1 if it intersects with a ray $\vec{r} \in R$ within laser sensor distance $r_{l}$, and 0 otherwise. The value of a cell $q_{o} \in Q_{\text {occluded }}^{t}$ is set to 1 if it is behind an obstacle cell $q_{i}$ and intersects with associated ray $\vec{r}$ in $Q_{o b s}^{\perp}$. This produces a "shadow" cast behind the obstacle extending to the edge of the grid. Formally, the value of each $q_{o} \in Q_{\text {occluded }}^{t}$ is given by

$$
v\left(q_{o}\right)= \begin{cases}1, & \exists\left(q_{i}, \vec{r}\right) \in Q_{o b s}^{\perp}: \vec{r} \perp q_{o}, \rho\left(q, q_{i}\right) \leq \rho\left(q, q_{o}\right) \\ 0, & \text { otherwise }\end{cases}
$$

where $\vec{r} \perp q_{o}$ indicates whether ray $\vec{r}$ intersects with cell $q_{o}$, and $\rho\left(q, q_{i}\right) \leq \rho\left(q, q_{o}\right)$ indicates whether the cell $q_{o}$ is behind the identified obstacle $q_{i}$.

Finally, the accumulated values in $Q_{\text {open }}$ are updated with the following:

$$
Q_{\text {open }} \leftarrow\left(Q_{\text {open }}+Q_{\text {open }}^{t} \delta\left(Q_{\text {occluded }}^{t}\right)\right) \delta\left(Q_{\text {obs }}\right)
$$

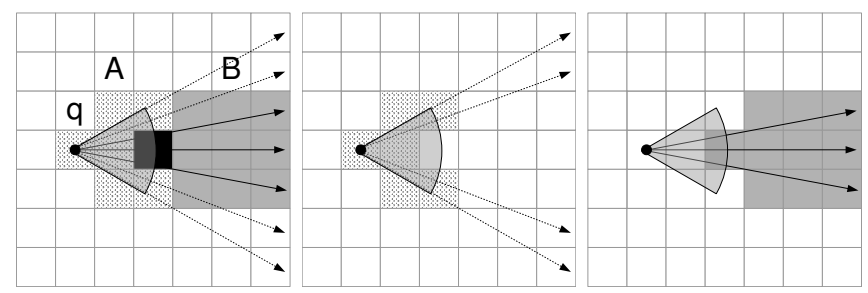

(a)

(b)

(c)

Fig. 2: In 2a, rays emanate from robot position $q$. Sensor range is indicated by shaded semicircle. The black space is an obstacle from the obstacle grid. Dotted spaces marked A are open, while gray spaces marked B are occluded. $2 \mathrm{~b}$ shows $Q_{\text {open }}$ with intersecting rays, and 2c shows $Q_{\text {occluded }}$ with intersecting rays.

This update allows us to accumulate a permanent, expanding open space during exploration. Any cells containing obstacles and newly discovered occluded space carved out. Similarly, the accumulated values in $Q_{\text {occluded }}$ are updated as follows:

$$
Q_{\text {occluded }} \leftarrow\left(Q_{\text {occluded }}+Q_{\text {occluded }}^{t}\right) \delta\left(Q_{\text {open }}\right)
$$

This update allows us to accumulate occluded space with newly discovered occluded areas, while eliminating any space which has been previously designated open or which as been visited before. A depiction of this process can be seen in Figure 2, with Figure 2a showing a scenario where a robot encounters an obstacle during exploration. Figures $2 \mathrm{~b}$ and $2 \mathrm{c}$ depict separate $Q_{\text {open }}$ and $Q_{\text {occluded }}$ grids, respectively.

\section{F. Sum of forces with occluded space}

Finally, we extend SBPF by incorporating the grid $Q_{\text {occluded }}$ into potential vector calculation, resulting in our O-SBPF approach. To model occluded space as a frontier by making its edges attractive, we introduce an additional grid $Q_{\text {frontier. }}$. For each $q_{i} \in Q_{\text {frontier }}, v\left(q_{i}\right)=1$ if $q_{i}$ is at the edge of occluded space, and 0 otherwise. $F_{O-S B P F}$ is given by the following extension to Equation 6:

$$
\begin{array}{r}
F_{O-S B P F}=w_{o b s} F_{\text {rep }}\left(q, Q_{\text {obs }}\right)+w_{\text {occ }} F_{\text {rep }}\left(q, Q_{o c c}\right) \\
+w_{\text {bot }} F_{\text {rep }}\left(q, Q_{\text {bot }}\right)+w_{\text {att }} F_{\text {att }}\left(q, \delta\left(Q_{o c c}\right) \delta\left(Q_{\text {occluded }}\right)\right) \\
+w_{\text {frontier }} F_{\text {att }}\left(q, \delta\left(Q_{\text {occ }}\right)\left(Q_{\text {frontier }}\right)\right)
\end{array}
$$

If $F_{O-S B P F}$ is used to determine robot heading and speed, the robot will be attracted to unexplored space and repulsed by previously visited space and obstacles. Occluded space will be neither attractive nor repulsive, though the edges of occluded space exert an attractive force to encourage robots to explore inside. As exploration continues, the repeated recomputation of $Q_{\text {occluded }}$ removes discovered occluded space from attractive potential calculation. Conversely, discovered open space is carved out of occluded space, allowing it to exert an attractive force again. 


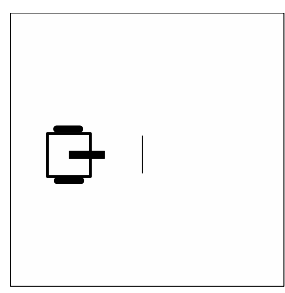

(a)

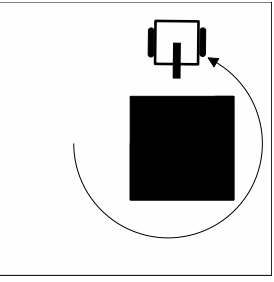

(b)

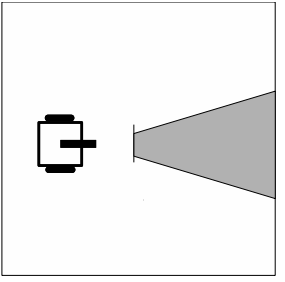

(c)
Fig. 3: SBPF and O-SBPF comparison. (a) and (b) show SBPF discovering a square obstacle by navigating around it, with repulsive space in black. (c) shows O-SBPF first encountering the obstacle, with occluded space in gray.

A simple illustration of O-SBPF can be seen in Figure 3. In SBPF, a robot must discover the entire enclosing boundary of an obstacle before it can be made repulsive. Figure 3 a shows a SBPF robot first encountering a square obstacle, while Figure $3 \mathrm{~b}$ shows the obstacle after the robot has fully explored its boundary. In contrast, O-SBPF can eliminate the space behind an obstacle without traveling around it, as shown in Figure 3c. Further exploration ultimately yields the same result as Figure $3 \mathrm{~b}$, as the obstacle is still filled once its enclosing boundary is discovered and occluded space is eliminated as it is found to be accessible. However, with O-SBPF, the robot is free to explore elsewhere with reduced attraction to the interior of the obstacle. In SBPF, the interior of the obstacle remains attractive in the time between Figures $3 a$ and $3 b$.

\section{EXPERIMENTS \& RESULTS}

In this section, we compare the SBPF algorithm with OSBPF, demonstrating the claims we proposed in Section 1:

1) Some rooms with inaccessible space cannot be fully explored with SBPF, but can be fully explored with O-SBPF.

2) Rooms with inaccessible space that can be fully explored with SBPF can be explored in equal or less time with O-SBPF.

Experiments were carried out in ROS Indigo [17] in the Stage environment, utilizing two simulated Turtlebot robots. Seven rooms maps were designed in a $25 \times 25$ grid. For each room, constants such as force multipliers, repulsive and attractive distance, and $k_{\text {att }}$ (equation 5) were tuned to find values which produced reasonable robot motion and consistent obstacle avoidance.

Fig. 4 depicts the room maps with initial robot positions and area bounds. In each room, we performed 20 simulation runs with robot movement determined by $F_{S B P F}$ and $F_{O-S B P F}$. To evaluate each run, we use percent coverage as the primary metric, representing the amount of space which robots visit over the course of the simulation. We consider a run successful if it reaches $95 \%$ coverage. In our simulations, robots either reached $95 \%$ or become stuck in a minima below

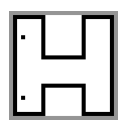

(a) $\mathrm{H}$

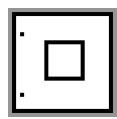

(b) Column

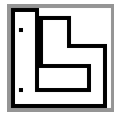

(c) Tunnel

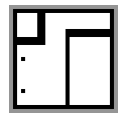

(f) Irregular

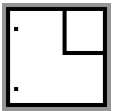

(d) Notch

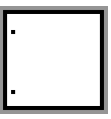

(g) Empty

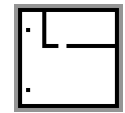

(e) Closet
Fig. 4: Room maps with robot starting points and area bounds

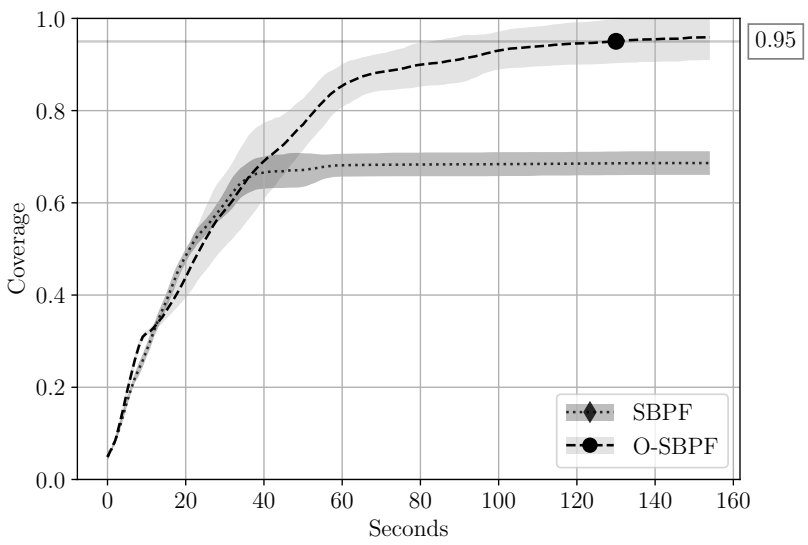

Fig. 5: Average coverage over time in the $\mathrm{H}$ room map. Shaded bands depict 1 standard deviation above and below the mean. $95 \%$ coverage is marked with with a diamond for SBPF and circle for O-SBPF.

this threshold, typically a cycle of attraction to inaccessible space and repulsion from the enclosing wall. Results of each simulation are summarized in Table 1.

Figures 5 and 6 show average coverage over time for the $\mathrm{H}$ and Column room. SBPF failed to overcome attraction to the large obstacles present in each room, becoming stuck in a minima at approximately $69 \%$ and $82 \%$ coverage for $\mathrm{H}$ and Column, respectively. In contrast, O-SBPF was able to avoid a minima by ignoring the empty space behind each obstacle. Consequently, O-SBPF achieved $95 \%$ coverage in an average of 130 and 109 seconds, significantly outperforming SBPF.

Figure 7 shows average coverage over time in the Tunnel room. In this room, O-SBPF and SBPF both reached $95 \%$ coverage at approximately 90 seconds, satisfying our condition that O-SBPF should not perform worse than SBPF. 


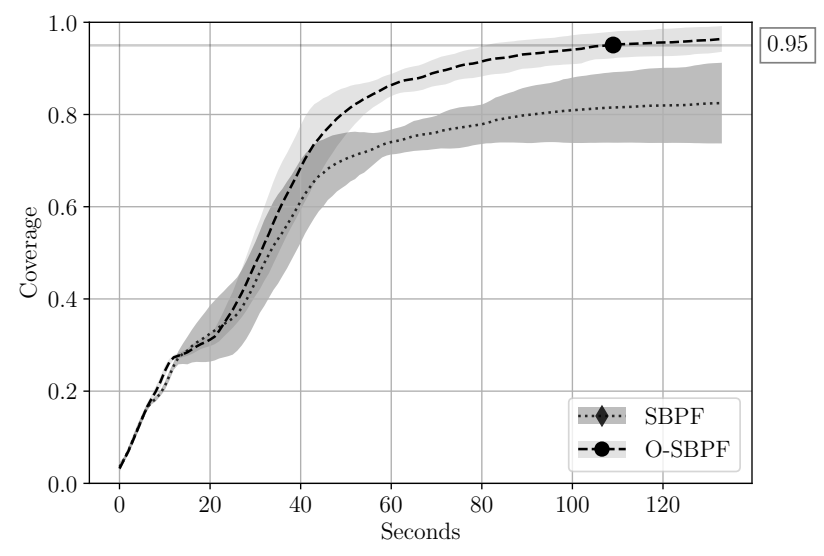

Fig. 6: Average coverage over time in the Column room map

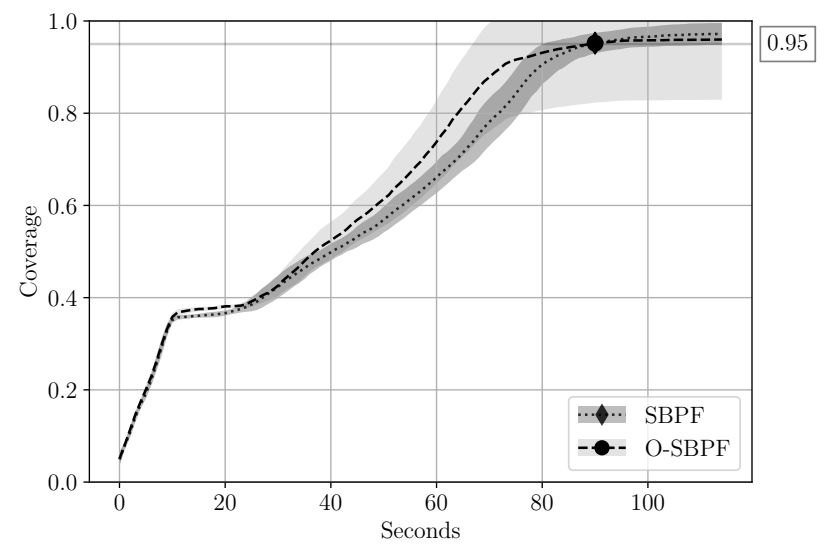

Fig. 7: Average coverage over time in the Tunnel room map

However, an examination of the graph reveals a tendency for O-SBPF to visit more space faster than SBPF earlier in exploration, with a wider standard deviation outperforming SBPF until approximately 90\% coverage at 75 seconds. Consequently, despite similar performance, we can say that O-SBPF slightly outperforms SBPF in early exploration in this room.

Figures 8 and 9 show results in the Notch and Closet rooms. Though each graph depicts similar performance between SBPF and O-SBPF for the duration of their runs, OSBPF still achieves 95\% coverage at 96 and 152 seconds before SBPF at 132 and 176 seconds, respectively. In Table 1 , we see the difference is significant for the Notch room with a $p$-value of 0.04 , and significant at $90 \%$ certainty for the Closet room with a $p$-value of 0.08 . Notably, Closet is one of the two rooms containing no inaccessible space, only space walled off with a narrow doorway permitting entry. Though O-SBPF eliminates the empty space inside the

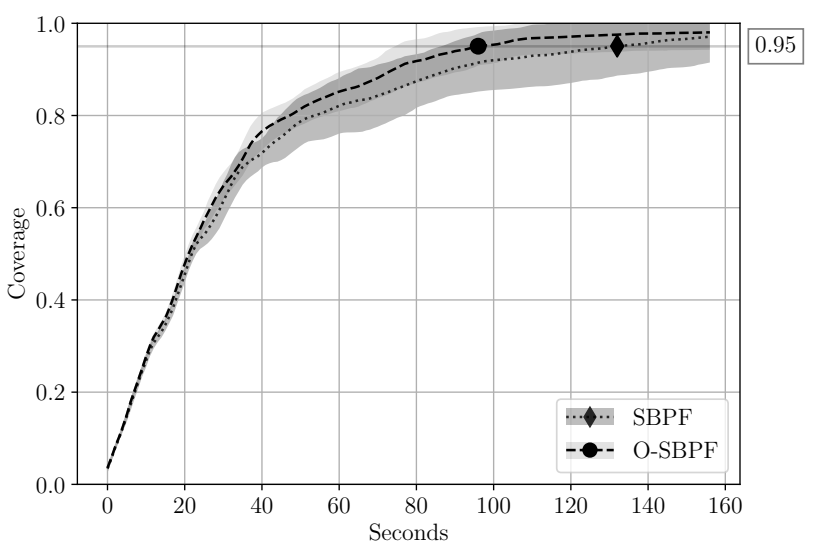

Fig. 8: Average coverage over time in the Notch room map

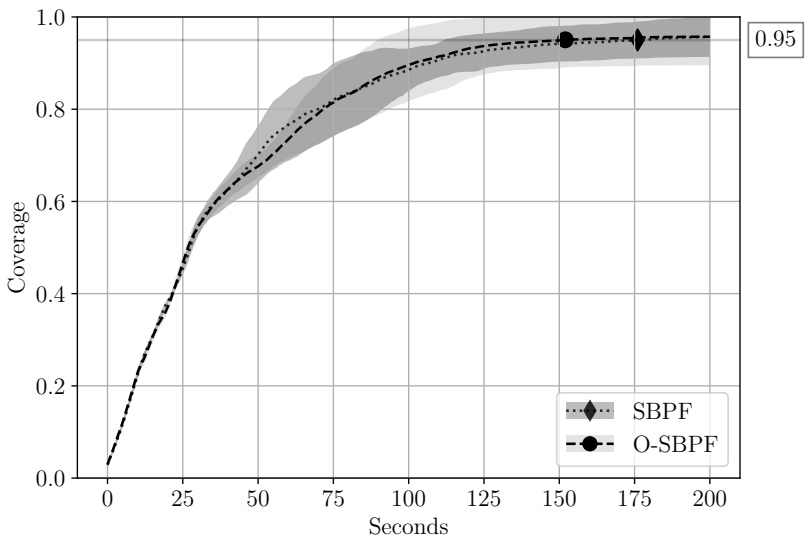

Fig. 9: Average coverage over time in the Closet room map

closet as the surrounding walls are discovered, the edges of unexplored space remain attractive as described in Section III-F. Consequently, the doorway into the closet remains attractive, permitting the robots to find their way inside.

Figures 10 and 11 show results in our final two rooms, Irregular and Empty. These rooms are unique in that SBPF and O-SBPF achieve 95\% coverage within seconds of one another, though with little difference between each run. In Irregular, SBPF finishes at 70 seconds slightly before O-SBPF at 75 seconds, though with a $p$-value of 0.10 . Consequently, we fail to reject the null hypothesis that SBPF finished no sooner than O-SBPF. In Empty, O-SBPF finishes at 93 seconds, slightly before SBPF at 98 seconds, though with a $p$-value of 0.43 . Similarly, we fail to reject the null hypothesis that O-SBPF finished no sooner than SBPF. We believe the similarity of these results can be partially explained by the layout of each room. For example, while Irregular contains corner obstacles opposite each other, the result is a narrowed 


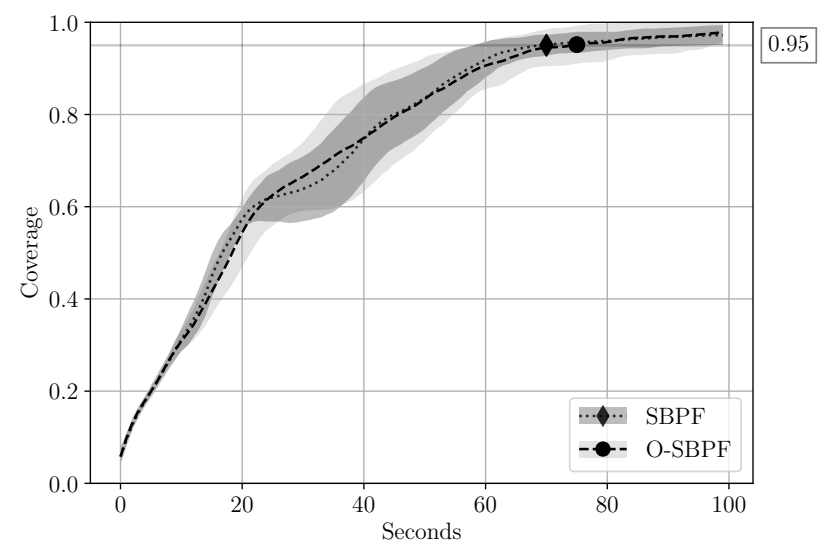

Fig. 10: Average coverage over time in the Irregular room map

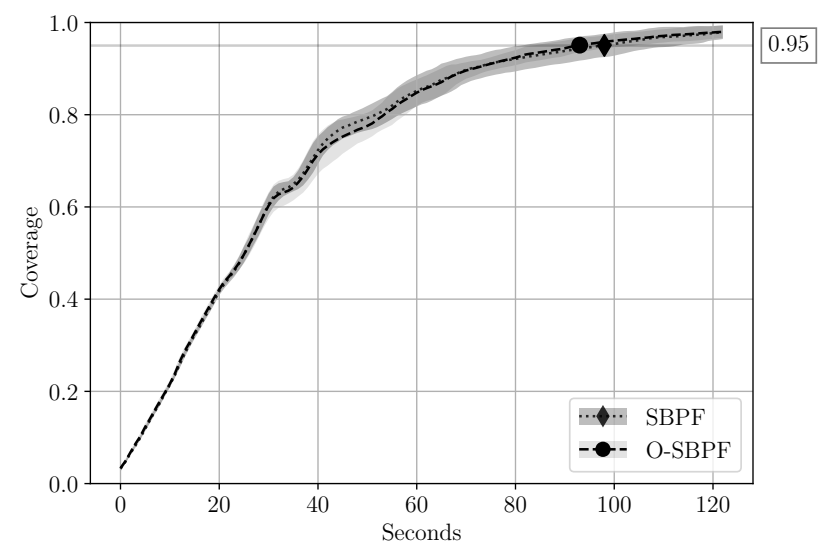

Fig. 11: Average coverage over time in the Empty room map

exploration space that could force robots to travel around each obstacle. The Empty room is a special case, as it was designed as a baseline with no obstacles at all. This is reflected in Figure 11, where there is little difference between SBPF and O-SBPF runs.

Out of all our results, those for $\mathrm{H}$ and Column were the most significant, demonstrating that O-SBPF can successfully navigate certain room types that SBPF alone cannot. We note that design elements of these rooms were distinct from other rooms: Column and $\mathrm{H}$ both feature large obstacles protruding deeply into exploration space, breaking up the flow of the room such that robots must follow different paths to explore the entire area. In contrast, simpler rooms such as Notch, Closet and Irregular featured less intrusive two-sided obstacles protruding into exploration space without posing any serious challenge to exploration. Even a relatively complex room such as Tunnel contains a fairly straightforward path for
TABLE I: Average time to reach 95\% coverage in seconds for all room maps. One-tailed $p$-value computed over time to reach $95 \%$ for each run. If a run did not reach $95 \%$ coverage, time was taken at the end of the run.

\begin{tabular}{lcccl} 
Room & SBPF & O-SBPF & $p$-value & Characteristics \\
\hline H & - & 130 & $1.11 \times 10^{-8}$ & Straight passage \\
Column & - & 109 & $8.92 \times 10^{-8}$ & Inaccessible center \\
Tunnel & 90 & 90 & 0.02 & Winding passage \\
Notch & 132 & 96 & 0.04 & Inaccessible corner \\
Closet & 176 & 152 & 0.08 & Accessible corner \\
Irregular & 70 & 75 & 0.10 & Inaccessible corners \\
Empty & 98 & 93 & 0.43 & Baseline
\end{tabular}

the robots to follow. This suggests that O-SBPF is especially suited for complex spaces with many obstacles, without any clear or straightforward path for the robots to follow. We discuss this as an opportunity for future experimentation in Section V.

\section{CONCLUSIONS}

The principal contribution of this paper is a novel approach to exploring unknown, enclosed spaces that improves upon the Space-Based Potential Field (SBPF) method by reducing attraction to inaccessible areas. SBPF addresses this issue by filling in inaccessible space as a solid obstacle once an enclosing boundary is discovered. We improved this technique by introducing O-SBPF and the novel space designations of open and occluded. These designations are used to produce a limited frontier in the potential field. To accomplish this, we described a ray-casting algorithm to designate points as open or occluded, and incorporated this designation into attractive force calculation to eliminate attraction to occluded space while introducing attraction to its boundary to encourage exploration.

Several simulations were run to demonstrate the effectiveness of O-SBPF, showing that O-SBPF performs as well as SBPF or better. Results were evaluated based on the time taken to reach $95 \%$ coverage. In two rooms with major obstacles, a large central column and multiple walled-off spaces protruding into the exploration space, SBPF became stuck in a minima well below 95\% coverage. In contrast, O-SBPF overcame attraction to the inaccessible space and achieved $95 \%$ coverage. In other rooms with simpler obstacles, O-SBPF generally achieved $95 \%$ coverage significantly earlier than SBPF, with only two failing to show significant difference between SBPF and O-SBPF. Of these, one was an empty space devoid of all obstacles, designed as a baseline. The other featured two obstacles on opposing corners of the space, which we hypothesize could have forced the robots along the relatively narrow spaces on either side.

As noted in Section IV, we found that some types of room were worse for SBPF than others. In this work, we performed a limited number of experiments in a set of simple rooms to establish the viability of O-SBPF. We believe O-SBPF 
will continue to significantly outperform SBPF in complex rooms, with large and varied obstacles that create complex exploration paths. Further experimentation in many different rooms designed around specific obstacle characteristics could establish quantifiable room types, as well as establish predictable performance characteristics for specific room types under both SBPF and O-SBPF.

While this work performed simulations in a ROS environment, the simulations were relatively simple and operated under ideal conditions. Further experimentation could bring simulations closer to real-world testing. For example, we used a centralized command server architecture for robot communication. Work by Liu and Lyons to decentralize SBPF [13] could be applied to O-SBPF, improving resilience and robot independence in poor communication conditions. Additionally, we did not utilize a Simultaneous Localization and Mapping (SLAM) system in our experiments, creating a simulated localization system instead. However, SLAM is crucial for real-world testing, since accurate location information might not be available in real-world testing scenarios. Finally, because O-SBPF was implemented in ROS in the Turtlebot environment, the same codebase can be used in real-world testing carried out in a room with physical obstacles.

\section{ACKNOWLEDGMENT}

We thank Rohan Agarwal for his valuable coding assistance in metrics collection, for contributing the Closet and Irregular room maps, and for carrying out robot experiments.

\section{REFERENCES}

[1] Francesco Amigoni, Jacopo Banfi, and Nicola Basilico. Multirobot exploration of communication-restricted environments: A survey. IEEE Intelligent Systems, 32(6):48-57, 2017.

[2] Torsten Andre and Christian Bettstetter. Collaboration in multi-robot exploration: to meet or not to meet? Journal of Intelligent \& Robotic Systems, 82(2):325-337, 2016.

[3] Ronald C Arkin. Behavior-based robotics. MIT press, 1998.

[4] Ronald C Arkin and Jonathan Diaz. Line-of-sight constrained exploration for reactive multiagent robotic teams. In 7th International Workshop on Advanced Motion Control. Proceedings (Cat. No. 02TH8623), pages 455-461. IEEE, 2002.

[5] Wolfram Burgard, Mark Moors, Dieter Fox, Reid Simmons, and Sebastian Thrun. Collaborative multi-robot exploration. In ICRA, pages 476-481, 2000 .

[6] Wolfram Burgard, Mark Moors, Cyrill Stachniss, and Frank E Schneider. Coordinated multi-robot exploration. IEEE Transactions on robotics, 21(3):376-386, 2005.

[7] Kyle Cesare, Ryan Skeele, Soo-Hyun Yoo, Yawei Zhang, and Geoffrey Hollinger. Multi-uav exploration with limited communication and battery. In Robotics and Automation (ICRA), 2015 IEEE International Conference on, pages 2230-2235. IEEE, 2015.
[8] Hao-Tien Chiang, Nick Malone, Kendra Lesser, Meeko Oishi, and Lydia Tapia. Path-guided artificial potential fields with stochastic reachable sets for motion planning in highly dynamic environments. In 2015 IEEE International Conference on Robotics and Automation (ICRA), pages 2347-2354. IEEE, 2015.

[9] Gregory Kahn, Peter Sujan, Sachin Patil, Shaunak Bopardikar, Julian Ryde, Ken Goldberg, and Pieter Abbeel. Active exploration using trajectory optimization for robotic grasping in the presence of occlusions. In Robotics and Automation (ICRA), 2015 IEEE International Conference on, pages 4783-4790. IEEE, 2015.

[10] Alina Kenealy, Nicholas Primiano, Alex Keyes, and Damian M Lyons. Thorough exploration of complex environments with a space-based potential field. In Intelligent Robots and Computer Vision XXXII: Algorithms and Techniques, volume 9406, page 940605. International Society for Optics and Photonics, 2015.

[11] Haye Lau. Behavioural approach for multi-robot exploration. In Australasian Conference on Robotics and Automation. Australian Robotics and Automation Association Inc, 2003.

[12] Marc Levoy. Display of surfaces from volume data. IEEE Computer graphics and Applications, (3):29-30, 1988.

[13] Tsung-Ming Liu and Damian M. Lyons. Leveraging area bounds information for autonomous decentralized multi-robot exploration. Robotics and Autonomous Systems, 74:66 - 78, 2015.

[14] Tsung-Ming Liu and Damian M Lyons. Leveraging area bounds information for autonomous multirobot exploration. In Intelligent Autonomous Systems 13, pages 563-576. Springer, 2016.

[15] Yuecheng Liu and Yongjia Zhao. A virtual-waypoint based artificial potential field method for uav path planning. In 2016 IEEE Chinese Guidance, Navigation and Control Conference (CGNCC), pages 949953. IEEE, 2016.

[16] Carlos Nieto-Granda, John G Rogers III, and Henrik I Christensen. Coordination strategies for multi-robot exploration and mapping. The International Journal of Robotics Research, 33(4):519-533, 2014.

[17] Morgan Quigley, Ken Conley, Brian Gerkey, Josh Faust, Tully Foote, Jeremy Leibs, Rob Wheeler, and Andrew Y Ng. Ros: an open-source robot operating system. In ICRA workshop on open source software, volume 3, page 5. Kobe, Japan, 2009.

[18] Alessandro Renzaglia and Agostino Martinelli. Potential field based approach for coordinate exploration with a multi-robot team. In 2010 IEEE International Workshop on Safety Security and Rescue Robotics (SSRR), pages 1-6. IEEE, 2010.

[19] Scott D Roth. Ray casting for modeling solids. Computer graphics and image processing, 18(2):109-144, 1982.

[20] Arnoud Visser, Julian De Hoog, Adrian Jiménez-González, and J$\mathrm{R}$ Martinez de Dios. Discussion of multi-robot exploration in communication-limited environments. In Workshop" Towards Fully Decentralized Multi-Robot Systems: Hardware, Software and Integration" at the ICRA Conference, 2013.

[21] Theeraphol Wattanavekin, Taiki Ogata, Tatsunori Hara, and Jun Ota. Mobile robot exploration by using environmental boundary information. ISRN Robotics, 2013, 2013.

[22] Brian Yamauchi et al. Frontier-based exploration using multiple robots. In Agents, volume 98, pages 47-53, 1998. 